\title{
One-year molecular survey of astrovirus infection in turkeys in Poland
}

\author{
Katarzyna Domanska-Blicharz • Anna Seroka • \\ Zenon Minta
}

Received: 29 October 2010/Accepted: 23 February 2011/Published online: 15 March 2011

(C) The Author(s) 2011. This article is published with open access at Springerlink.com

\begin{abstract}
The presence of turkey astrovirus (TAstV) was monitored in meat-type turkey flocks in Poland in 2008. Clinical samples (10 individual faecal swabs/flock) from 77 flocks aged 1-19 weeks were collected from different regions of the country. RT-PCR experiments were performed for detection and molecular characterization of TAstV using four sets of primers within the RdRp gene (ORF1b). The prevalence of astrovirus was 34/77 (44.15\%) in the flocks tested. TAstV type 2 was associated with 30 of 77 infections (38.9\%), either alone or in mixed infections; $\mathrm{TAst} V$ type 1 was detected in 9 of 77 flocks (11.6\%), either alone or in mixed infections; ANV was detected only in one flock $(1.29 \%)$ by sequence analysis during this study. Phylogenetic analysis revealed genetic variability in the TAstV strains that were isolated. Some of Polish TAstV-2 strains were genetically related to the North American isolates; however, most of them formed a distinct subgroup of "European" isolates, suggesting their separate origin or evolution. Additionally, due to the high variability of the $\mathrm{TAst} V$ sequences, the most suitable method for TAstV typing seems to be sequencing.
\end{abstract}

\section{Introduction}

The history of studies on astroviruses (AstV) is rather short, as they were first described by Appleton and Higgins in 1975 in the context of gastrointestinal problems in

K. Domanska-Blicharz $(\bowtie) \cdot$ A. Seroka $\cdot$ Z. Minta

Department of Poultry Diseases, National Veterinary Research Institute, Al. Partyzantow 57, 24-100 Pulawy, Poland

e-mail: domanska@piwet.pulawy.pl children [1]. Subsequently identified astroviruses were also associated with enteritis and affected a wide range of host species, such as lambs, calves, piglets, dogs, turkey poults, chickens, guinea fowl, red and roe deer, cats, mice, and mink $[2,4,14,16,26]$. Additionally, some astroviruses have also been associated with non-intestinal illness. In ducklings, the virus known as duck virus hepatitis type 2 (DVH2) causes fatal hepatitis, and in chickens, avian nephritis virus (ANV) results in mild growth depression, kidney lesions and mortality $[8,10]$.

The effects of enteric diseases are especially visible in the intensive poultry industry. The consequences, such as decreased weight gain, increased morbidity and mortality, poor feed conversion and increased use of therapeutic antimicrobial treatments, all contribute to important economic losses. Different terms have been used to describe enteric disease syndrome: runting-stunting syndrome of broilers (RSS), poult enteritis complex (PEC), and poult enteritis mortality syndrome (PEMS). However, none of these descriptions relates to the specific infectious agents, and numerous viruses have been associated with them. However, astroviruses seem to be the most frequently involved in enteritis $[2,8,12,13,24]$. Among five different astroviruses identified in avian species, three types have been detected in turkeys. Diarrhoea and increased mortality in turkey poults associated with the presence of an astrovirus, now called turkey astrovirus type 1 (TAstV-1), were described for the first time in 1980 in the United Kingdom [16] and, five years later, in the USA [24, 25]. Turkey astrovirus type 2 (TAstV-2), which is antigenically and genetically distinct from the previously identified TAstV-1, was isolated in the USA in 1996 [13]. With regard to ANV, initially antibodies against this virus were only found in turkey flocks in the UK and Japan [17, 28]. Subsequently, the virus was detected using molecular techniques in 
intestinal contents of healthy and diseased poults in the USA, but its prevalence is low [5, 20, 21].

Astroviruses are small, round, non-enveloped particles, typically $28-30 \mathrm{~nm}$ in diameter, having a star-like appearance in electron microscopy. AstV has a 6.8-7.9-kb positive-sense single-stranded RNA genome, which encodes three open reading frames (ORF1a and ORF1b, which are linked by a translational frameshift, and ORF2). ORF1b encodes the RNA-dependent RNA polymerase, and ORF2 encodes the capsid protein $[3,7,11]$. The ORF1b region is the most conserved gene in mammalian and avian astroviruses as well as in avian astroviruses [13]. On the other hand, ORF2 is the most variable region of the astrovirus genome and is possibly responsible for the antigenic and pathogenic diversity of astrovirus strains [23]. The family Astroviridae is divided into two genera: Mamastrovirus (mammalian astrovirus) and Avastrovirus (avian astrovirus). Recently, the Astroviridae Study Group of the International Committee for Taxonomy of Viruses (ICTV) has proposed a classification system based on genetic criteria instead of the host of origin. The genus Mamastrovirus includes19 astrovirus genotypes, and the genus Avastrovirus includes three genotypes that are distinguished based on the amino acid sequence of full-length ORF2 (http:// talk.ictvonline.org/files/proposals/taxonomy_proposals_ vertebrate $1 / \mathrm{m} /$ vert01/2358.aspx).

The aim of the present study was to investigate the prevalence of TAstV infections in commercial meat-type turkey flocks in Poland and to estimate their genetic relatedness, based on the ORF1b region, to those available in the web database.

\section{Materials and methods}

Swab samples

In 2008, a total of 770 fecal swabs were collected from 77 turkey flocks (10 individual faecal swabs/flock) located in different regions of Poland. Samples were collected from poults aged 1 to 19 weeks. Most of the flocks tested showed one or more of the enteritis symptoms, most frequently diarrhea, thermoregulatory disorders, depression and growth retardation. In 20 flocks, the birds were in good health, while PEMS symptoms (with increased mortality) were observed in 10 flocks. All samples were stored below $-65^{\circ} \mathrm{C}$ until processing. After slow thawing, each individual swab was hydrated in phosphate-buffered saline (PBS) supplemented with antibiotics (100 U of penicillin with $100 \mathrm{mg}$ of streptomycin/ml), incubated for $1 \mathrm{~h}$ at room temperature, clarified by centrifugation at $1500 \mathrm{~g}$ for 20 min and used for RNA extraction.

\section{RNA extraction}

Total RNA was extracted from $250 \mu$ of supernatant from 5 pooled swabs ( 2 pools/flock) using an RNeasy Mini Kit (QIAGEN, Germany) according to the manufacturer's instructions. Each RNA was eluted in $50 \mu \mathrm{l}$ RNase-free water.

Molecular methods

Four different RT-PCR assays directed toward various regions of ORF1b were used for astrovirus detection and differentiation. Conventional RT-PCR was performed using a QIAGEN OneStep RT-PCR kit (QIAGEN, Germany), and the products were separated on a $2 \%$ agarose gel in Tris-acetate-EDTA buffer and visualized using ethidium bromide stain. Real-time RT-PCR was performed using a QuantiTect Probe RT-PCR Kit (QIAGEN, Germany) in a 7500 Real Time PCR System (Applied Biosystems, USA).

Nucleotide sequencing and phylogenetic analysis

Amplicons $601 \mathrm{bp}$ in length were obtained in the RT-PCR assay aimed at detecting all TAstV types. Amplicons were purified using a NucleoSpin Extract II Kit (Macherey Nagel, Germany) according to the manufacturer's instructions and sequenced at the commercial service Genomed (Poland). Sequencing was performed in both directions with the same primers that were used for RT-PCR reactions. Using the Seqman program (DNASTAR, Madison, WI), the forward and reverse nt sequences were edited, cured and aligned as one consensus sequence. Multiple alignments of nt and aa sequences were performed using the MegAlign application (DNASTAR, Madison, WI) using the Clustal W method. Phylogenetic analysis of aligned sequences was performed with MEGA 4.1 using the neighbor-joining method with the maximum-likelihood model. Bootstrap scores were generated from 1000 replicates. The nt sequences were translated to deduced amino acid (aa) sequences, which were also compared to detect any changes at the aa level.

Nucleotide sequence accession numbers

The astrovirus strains used were as follows: AR/CK/ SEP-A802/05 (DQ324835), Brazil/CK/USP336-1/09 (GU 585493), Brazil/TK/USP351-1a/09 (GU938474), CA/TK/ SEP-A270/04 (DQ066570), CA/TK/SEP-A273/04 (DQ 066578), CA/CK/SEP-A779/05 (DQ453922), DE/CK/SEPA651/05 (DQ324847), France/GF1806 (EU165343), France/ GF/2006 (EU165345), A/CK/SEP-A367/05 (DQ324827), GA/CK/SEP-A451/05 (DQ324828), Italy/GF/766/01 
(DQ381379), Italy/GF/1716/04 (DQ381396), Italy/TK/89/ 04 (DQ381365), Italy/TK/100/04 (DQ381284), Italy/TK/ 639/04 (DQ381368), Italy/TK/1047/04 (DQ381324), Italy/ TK/1619/04 (DQ381372), Italy/TK/1820/04 (DQ381375), Italy/TK/2654/04 (DQ381378), Italy/TK/6363-4/04 (DQ 381376), Japan/CK/G-4260 (AB033998), MN/TK/B1002/08 (FJ605155), MN/TK/B2011/08 (FJ693665), MN/TK/B3027/ 08 (FJ693683), MN/TK/SEP-A805/05 (DQ324814), MO/ TK/SEP-A815/05 (DQ324817), MO/TK/SEP-A819/05 (DQ 324824), MO/TK/SEP-A827/05 (DQ324819), NC/TK/SEPA41/03 (DQ066580), NC/TK/SEP-A306/04 (DQ324809), NC/TK/SEP-A498/05 (DQ324821), NC/TK/SEP-A537 (DQ 324837), NC/TK/SEP-A552/05 (DQ324823), NC/TK/SEPA670/05 (DQ324838), OH/TK/SEP-A848/1986 (DQ 324820), South Africa/CK/612/1994 (EU669001), TAstV-1 (Y15936), TAstV/AK/98 (EU143843), TAstV/CA/00 (EU 143844), TX/TK/SEP-A312/04 (DQ066594), United Kingdom/CK/EF91-272/1984 (EU669002), VA/TK/SEP-A226/ 03 (DQ066596).

Sequences determined in this study have been submitted to GenBank with accession numbers from HQ317705 to HQ317733.

\section{Results}

TAstV typing by RT-PCR techniques

Fecal swabs obtained from all 77 turkey flocks were tested for the presence of RNA of any type of TAstV. RNA of turkey astrovirus was detected in swabs from 34 flocks (44\%). For differentiation between different types of turkey astrovirus, three further tests were performed with published primers targeting various type-specific regions of the polymerase gene (Table 1). Mixed infection with TAstV-1 and TAstV-2 was observed in swabs from six flocks (17.6\%), and TAstV-2 was detected in 20 flocks (58.8\%).
However, in eight flocks, no TAstV was detected by RTPCR $(23.5 \%)$. All flocks were negative for ANV by this technique (Table 2).

Genetic analysis of turkey astroviruses

The 601-bp amplicons obtained by RT-PCR with primers TAPG-L1 and TAPG-R1 from 29 TAstV-positive flocks were sequenced. In eight cases where RT-PCR was unsuccessful, sequencing showed that three samples contained TAstV-1 (flocks 4, 5, 31), four contained TAstV-2 (flock 8, 10, 18, 20) and one contained ANV (flock 21). Of the six flocks in which RT-PCR showed coinfection with $\mathrm{TAstV}-1$ and 2, sequencing experiments indicated that three of them were infected with TAstV-1 (flock 9, 14, 16), and the others were infected with TAstV-2 (flocks 7, 11, 33). Fifteen flocks tested positive for TAstV-2 by RT-PCR, and this was also confirmed by sequence analysis (Table 2).

Polish avian astrovirus isolates are divided into three groups (Fig. 1): TAstV-1-like, TAstV-2-like and ANVlike. Most of them belong to the TAstV-2 type. These isolates formed two subgroups (subgroups 1 and 2), with the majority being in subgroup 2 (17 isolates). In subgroup 1 , three subclades $(\mathrm{a}, \mathrm{b}, \mathrm{c})$ could be differentiated, and all six Polish isolates clustered in one subclade (a) together with astroviruses previously reported from the USA. Subclade $b$ included strains from the USA, Italy and France that were isolated either from turkey or guinea fowl; however, the latter formed separate sublineages. Subclade $\mathrm{c}$ included strains only from the USA. A comparison of nucleotide and amino acid sequences of six Polish turkey astrovirus type 2 strains from subgroup 1, showed $93.5-99.5 \%$ and $96.8-98.9 \%$ similarity to each other and 86.9-91.2 and $93.5-96.8 \%$ to the prototype TAstV-2 isolate CA/00. Subgroup 2 TAstVs did not show any distinct subclades and included only European isolates

Table 1 Primers and probe used in this study

\begin{tabular}{|c|c|c|c|c|c|}
\hline Primer & Location & Sequence & Target & Amplicon (bp) & Ref \\
\hline TAPG-L1 & $3835-3853^{\mathrm{A}}$ & TGGTGGTGYTTYCTCAARA & \multirow{2}{*}{$\begin{array}{l}\text { All } \\
\text { TAstV }\end{array}$} & \multirow[t]{2}{*}{601} & \multirow[t]{2}{*}[29]{} \\
\hline TAPG-R1 & $4414-4432^{\mathrm{A}}$ & GYCKGTCATCMCCRTARCA & & & \\
\hline T1pol1F & $4133-4156^{\mathrm{A}}$ & AGCTyATGmGGTTCTTTCTTCTyG & \multirow[t]{2}{*}{ TAstV-1 } & \multirow[t]{2}{*}{251} & \multirow[t]{2}{*}[5]{} \\
\hline T1pol1R & $4360-4383^{\mathrm{A}}$ & GATGGTGGGTAGCCTATTGTGTTC & & & \\
\hline ANVpol1F & $4097-4118^{\mathrm{B}}$ & GyTGGGCGCyTCyTTTGAyAC & \multirow[t]{2}{*}{ ANV } & \multirow[t]{2}{*}{473} & \multirow[t]{2}{*}[5]{} \\
\hline ANVpol1R & $3644-3665^{\mathrm{B}}$ & CrTTTGCCCkrTArTCTTTrT & & & \\
\hline TAV4248F & $4248-4268^{\mathrm{C}}$ & TCCTCCATGATTCTCATAAG & \multirow[t]{3}{*}{ TAstV-2 } & \multirow[t]{3}{*}{112} & \multirow[t]{3}{*}[27]{} \\
\hline TAV4360F & $4344-4360^{\mathrm{C}}$ & CTTGACCTGGCAAACT & & & \\
\hline TAV4274 PB & $4274-4291^{\mathrm{C}}$ & Fam-AAGATGCGGCGCTTGTA-Tamra & & & \\
\hline
\end{tabular}

Primer or/and probe positions refer to the sequences for ${ }^{\mathrm{A}}$ TAstV-1 (GenBank accession no. Y15936), ${ }^{\text {B }}$ ANV Japan/CK/G-4260 (GenBank accession no. AB033998) and ${ }^{\mathrm{C}}$ TAstV-2 strain TAstV/CA/00 (GenBank accession no. EU143844) 
Table 2 Results of studies on Polish turkey astrovirus

\begin{tabular}{|c|c|c|c|c|c|}
\hline & Isolate & $\begin{array}{l}\text { Condition } \\
\text { of animals }\end{array}$ & Age & $\begin{array}{l}\text { RT-PCR } \\
\text { typing }\end{array}$ & $\begin{array}{l}\text { Sequence-determined } \\
\text { typing }\end{array}$ \\
\hline 1 & PL/TK/G56/08 & PEC & 10 weeks & TAstV-2 & - \\
\hline 2 & PL/TK/G59/08 & PEMS & 5 weeks & TAstV-2 & - \\
\hline 3 & PL/TK/79/08 & PEMS & 9 days & TAstV-2 & TAstV-2 \\
\hline 4 & PL/TK/81/08 & PEC & 12 weeks & No & TAstV-1 \\
\hline 5 & PL/TK/85/08 & PEC & 5 weeks & No & TAstV-1 \\
\hline 6 & PL/TK/92/08 & PEC & 4 weeks & TAstV-2 & TAstV-2 \\
\hline 7 & PL/TK/G108/08 & healthy & 3 weeks & TAstV-1, TAstV-2 & TAstV-2 \\
\hline 8 & PL/TK/G109/08 & PEC & 4 weeks & No & TAstV-2 \\
\hline 9 & PL/TK/G110/08 & PEC & 16 days & TAstV-1, TAstV-2 & TAstV-1 \\
\hline 10 & PL/TK/G114/08A & healthy & 7 weeks & No & TAstV-2 \\
\hline 11 & PL/TK/G116/08 & PEMS & 3 weeks & TAstV-1, TAstV-2 & TAstV-2 \\
\hline 12 & PL/TK/G118/08 & PEMS & 25 days & TAstV-2 & TAstV-2 \\
\hline 13 & PL/TK/G121/08 & healthy & 7 weeks & TAstV-2 & - \\
\hline 14 & PL/TK/G122/08 & healthy & 5 weeks & TAstV-1, TAstV-2 & TAstV-1 \\
\hline 15 & PL/TK/G123/08 & healthy & 9 weeks & TAstV-2 & TAstV-2 \\
\hline 16 & PL/TK/G125/08 & healthy & 6 weeks & TAstV-1, TAstV-2 & TAstV-1 \\
\hline 17 & PL/TK/G126/08 & healthy & 4 weeks & TAstV-2 & TAstV-2 \\
\hline 18 & PL/TK/G130/08 & $\mathrm{n} / \mathrm{a}$ & $\mathrm{n} / \mathrm{a}$ & No & TAstV-2 \\
\hline 19 & PL/TK/G143/08 & PEC & 8 days & TAstV-2 & TAstV-2 \\
\hline 20 & PL/TK/G144/08 & PEC & 10 weeks & No & TAstV-2 \\
\hline 21 & PL/TK/G145/08 & PEMS & 4 weeks & No & ANV \\
\hline 22 & PL/TK/G164/08 & healthy & 4 weeks & TAstV-2 & - \\
\hline 23 & PL/TK/G165/08 & PEMS & 7 days & TAstV-2 & TAstV-2 \\
\hline 24 & PL/TK/G182/08 & PEC & 2 weeks & TAstV-2 & TAstV-2 \\
\hline 25 & PL/TK/G188/08 & PEMS & 5 weeks & TAstV-2 & TAstV-2 \\
\hline 26 & PL/TK/G190/08 & PEMS & 14 weeks & TAstV-2 & TAstV-2 \\
\hline 27 & PL/TK/G193/08-K1 & PEC & 3 weeks & TAstV-2 & TAstV-2 \\
\hline 28 & PL/TK/G193/08-K3 & PEC & 2 weeks & TAstV-2 & TAstV-2 \\
\hline 29 & PL/TK/G193/08-K4 & PEC & 4 weeks & TAstV-2 & TAstV-2 \\
\hline 30 & PL/TK/G193/08-K5 & PEC & 2 weeks & TAstV-2 & TAstV-2 \\
\hline 31 & PL/TK/G197/08 & PEC & 4 weeks & No & TAstV-1 \\
\hline 32 & PL/TK/G213/08 & PEC & 3 weeks & TAstV-2 & TAstV-2 \\
\hline 33 & PL/TK/G222/08 & PEC & 2 weeks & TAstV-1, TAstV-2 & TAstV-2 \\
\hline 34 & PL/TK/G223/08 & PEMS & 23 days & TAstV-2 & TAstV-2 \\
\hline
\end{tabular}

No no result; PEC (poult enteritis complex) diarrhea, thermoregulation problems, depression, huddle; PEMS (poult enteritis mortality syndrome) same as above, and additionally, acute enteritis, increased mortality; $n / a$ not available

from Poland and Italy. Comparison of a 366-nucleotide region of ORF1b (from nt 4136 to 4502 of the TAstV-2 genome) revealed that, among these isolates, nt identity was between 96.9 and $100 \%$, and deduced amino acid identity was between $97.6 \%$ and $100 \%$. In turn, nucleotide and amino acid similarity of this subgroup to the prototype TAstV-2 was $89.5-90.6 \%$ and $95.3-96.5 \%$, respectively. Indeed, these isolates formed a separate subgroup, but its distinction was rather weakly supported statistically (bootstrap value 60).
Among the five Polish TAstV-1 isolates, nucleotide identity was $97.4-99.8 \%$, and amino acid identity was 98.2$100 \%$. They were also closely related to the prototype TAstV-1 isolate, with identities of $95-95.5 \%$ at the nucleotide level and 98.2-98.8\% at the amino acid level. The group of TAstV-1-like isolates was rather homogenous and included isolates from Poland, Brazil and the USA.

The nucleotide and amino acid identity between the Polish turkey ANV isolate and the ANV prototype Japan/ CK/G-4260 isolate was $86.7 \%$ and $97.1 \%$, respectively. In 
Fig. 1 Phylogenetic tree of nucleotide sequences of the ORF1b gene of TAstV.

Sequences are identified by country/origin/code/year and are described in "Materials and methods". Sequences indicated by a black dot are from the present study, and those that are underlined are strains that were used as references. The

phylogenetic tree was

constructed using the neighborjoining algorithm and the maximum-likelihood model with 1000 bootstrap replicates (bootstrap values shown on tree)

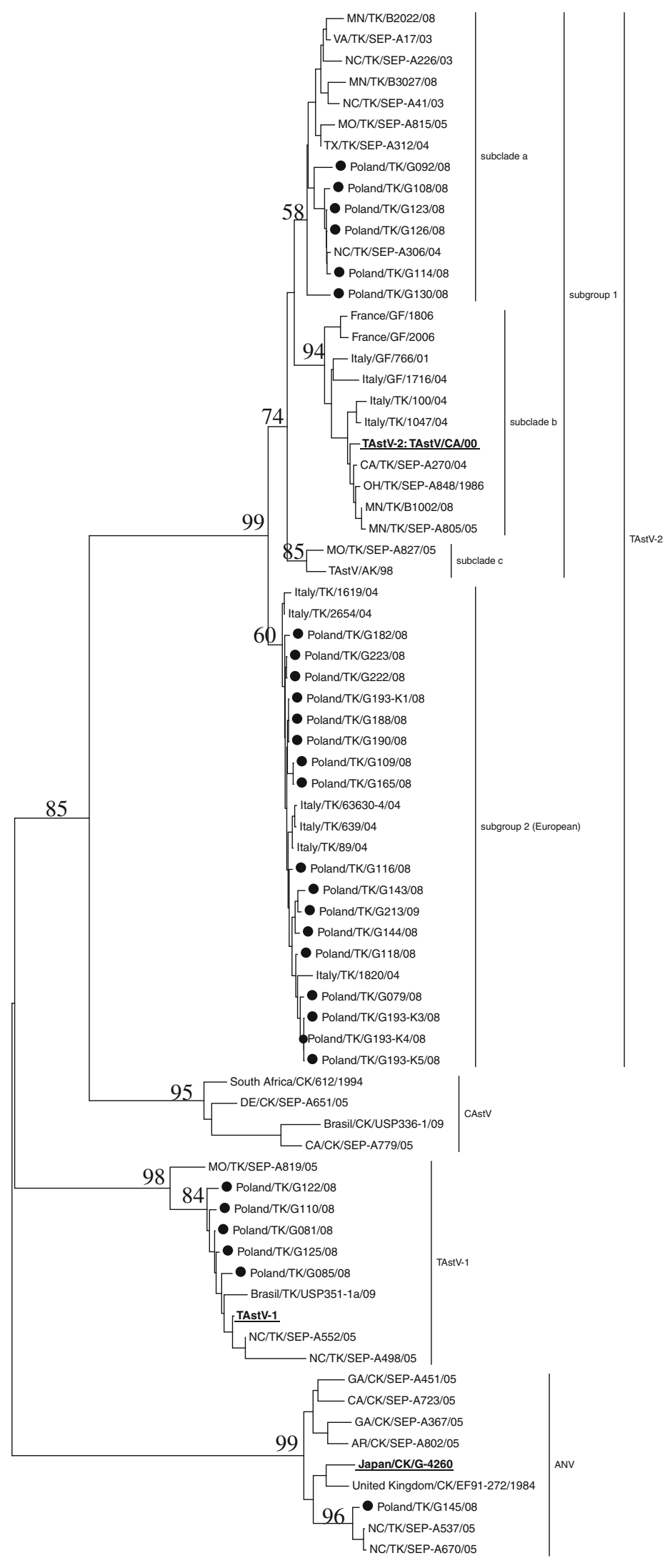


the group of ANV-like viruses, four subclades were identified. The only ANV-like virus detected in Poland clustered with ANVs isolated from turkey flocks in North Carolina in 2005. The other subclades included only viruses from chickens; however, it appears that the isolates from turkeys were more closely related to strains isolated from chickens in Japan and the UK than those from the USA.

\section{Discussion}

As with other poultry diseases, many molecular methods are used for diagnosis of etiological factors in enteric diseases of birds. For detection of chicken and turkey astroviruses, RT-PCR-based methods have been developed that target conserved regions of ORFs1b and ORF2, which encode the viral polymerase and capsid protein, respectively $[4,5,29,30]$. Because mixed infections with various types of astrovirus were identified in some cases, there was a need to distinguish between them. At first, the differentiation of four types of astroviruses, TAstV-1, TAstV-2, CAstV and ANV, relied on sequencing the amplicons. More recently, a new multiplex RT-PCR assay with primers specific for each of the four types of astroviruses was developed and used for survey of enteric viruses in the USA [5]. Overall, this method was unsuitable for differentiation of Polish isolates of astrovirus. Some isolates had additional changes in their nt sequences that prevented correct identification of astrovirus types (e.g., in the ORF1b sequence of PL/TK/G085/08, targeted by the T1pol1F primer, next to the three degenerate nucleotides, there were three additional nt mismatches; in the ORF1b sequence of PL/TK/G145/08 targeted by the ANVpolF primer, next to the four degenerate nucleotides, there were two additional nt changes). On the other hand, the usefulness of the primer set developed by Tang et al. [29] for detection of astrovirus seems to have been confirmed, as it was possible to detect astroviruses with many sequence variations.

The presence of astrovirus in 44.15\% (34/77) of Polish turkey flocks studied during 2008 is reported. The most frequently identified was TAstV-2, with samples positive from 30 of 77 flocks (38.9\%), either as single or mixed infections. TAstV-1 was detected in only nine flocks, either as single or mixed infections (11.6). ANV was detected in one flock $(1.29 \%)$. These figures indicate that the prevalence of TAstVs in Poland was lower when compared with a prevalence of $84 \%$ reported in commercial turkey flocks during a survey in Minnesota, USA, during 2007-2008 by Jindal et al., or of 59\%, reported in the UK; however, the prevalence in Poland was comparable to reports from Brazil (41.1\%), as reported by Villarreal et al. [9, 12, 32]. However, it should be noted that in all the previous studies, astrovirus detection was focused only on the TAstV-2 type.
In another study from the USA, astrovirus was detected in $100 \%$ of the turkey flocks tested, among which TAstV-2 was the most frequently identified $(69.6 \%)$, followed by TAstV-1 (28\%) and ANV (12.5\%) [21].

Astroviruses were mainly detected in flocks suffering from enteric disorders; however, a few were detected in flocks without any symptoms of disease. This is in accordance with previous findings, and to date, the factors influencing astrovirus pathogenicity are unknown $[22,31]$. Astrovirus was seldom detected in flocks older than 4 weeks. Recently, Jindal et al. observed astroviruses in poults up to 9 weeks of age [12]. In Poland, the maximum age of astrovirus-positive poults was 14 weeks, suggesting long-lasting astrovirus infection in turkeys or susceptibility to infection among older poults.

TAstV-1 was first described in 1980 and mainly detected in the USA [18]. Recently, the presence of TAstV-1 was also confirmed in Brazil (data based on available sequences in GenBank: TAst-1/Brazil/2009/USP350-3B: GU938473, TAst-1/Brazil/2009/USP351-1A: GU938474). Regarding ANV, at first, only antibodies against ANV were detected in turkeys in Japan and the UK, and the first isolation and sequencing was achieved in 2006 in the USA $[6,17,18]$. In our study, the presence of an ANV-like isolate in a Polish turkey flock was detected, and this is, to the authors' knowledge, the first time this virus has been detected outside the USA.

Genetic variability among astroviruses was observed, and this confirms previous results from the USA and Europe $[5,18,19]$. For ORF1b of TAstV-2, two subgroups could be distinguished. In subgroup 1, three subclades were identified. All six Polish TAstV-2 isolates clustered in subclade a together with North American isolates of group 1 identified by Pantin-Jackwood at al. and astroviruses from group 2 identified by Jindal at al. [4, 12, 18]. Subclade $b$ included North American isolates from group 2 identified by Pantin-Jackwood et al. and from group 1 identified by Jindal et al. as well as TAstV from Italian group G1. In our comparison, AstV isolates from guinea fowl from Italy that were previously assigned to the G3 group and isolates from France (sequences available in GenBank) also clustered in subclade b, but in separate sublineages. Subgroup 2 included only European isolates originating from Italy (isolated in 2004/05) and Poland (2008). Italian isolates from this subgroup were previously described as a distinct genetic lineage, G2 [4]. A similar subgrouping of European and "American-like" isolates of TAstV-2 was previously indicated by Maurel at al. [15]. However, the comparison of sequences of Polish and French TAstV strains was not possible, since the sequences were not available in the public domain.

The results of phylogenetic studies have suggested a separate origin/evolution of European and North-American 
isolates. Taking into account that the ORF1b region, encoding RNA-dependent RNA polymerase, is the most conserved in the astrovirus genome and seems to vary according to geography, the presence of European isolates in "American" subgroup 1 might be the result of international/continental commercial exchange of birds [19].

In this study, we identified three types of astroviruses, TAstV-1-like, TAstV-2-like and ANV-like, circulating in turkey flocks in Poland. The most frequently identified were TAstV-2, with TAstV-1 occurring rarely and ANV occasionally. Isolated astroviruses were found to be genetically variable based on the sequence analysis reported here. This diversification was particularly noticeable among Polish TAstV-2 strains, as some of them were genetically related to the North American isolates. However, most of them formed a distinct subgroup of "European" isolates, suggesting their separate origin or evolution. In spite of the fact that some isolates were from healthy flocks, it could not be ruled out that astroviruses caused the observed health problems and poor performance of meat-type turkey flocks in Poland.

It should be also mentioned that in the light of the new classification rules, none of the Polish turkey astroviruses studied here could be assigned to any of the genotypes. In order to enable classification of these viruses, a phylogenetic analysis of the full-length ORF2 gene is needed, and according to the new taxonomy proposal, they are classified as " related viruses that might be members of the genus Avastrovirus but have not been assigned to a species" [23].

The results presented here also have some diagnostic implications. The conventional RT-PCR method developed by Tang et al. seems to be useful for detection of all TAstV infections, but additional methods for differentiation between astrovirus types failed to distinguish between them. For this purpose, the most suitable method still is sequencing. On the other hand, methods for differentiation of astrovirus types might reveal mixed astrovirus infections.

Open Access This article is distributed under the terms of the Creative Commons Attribution Noncommercial License which permits any noncommercial use, distribution, and reproduction in any medium, provided the original author(s) and source are credited.

\section{References}

1. Appleton H, Higgins PG (1975) Letter: viruses and gastroenteritis in infants. Lancet 1:1297

2. Baxendale W, Mebatsion T (2004) The isolation and characterisation of astroviruses from chickens. Avian Pathol 33:364-370

3. Carter MJ, Willcocks MM (1996) The molecular biology of astroviruses. Arch Virol Suppl 12:277-285
4. Cattoli G, De Battisti C, Toffan A, Salviato A, Lavazza A, Cerioli M, Capua I (2007) Co-circulation of distinct genetic lineages of astroviruses in turkeys and guinea fowl. Arch Virol 152: 595-602

5. Day JM, Spackman E, Pantin-Jackwood M (2007) A multiplex RT-PCR test for the differential identification of turkey astrovirus type 1 , turkey astrovirus type 2, chicken astrovirus, avian nephritis virus, and avian rotavirus. Avian Dis 51:681-684

6. Frazier JA, Howes K, Reece RL, Kidd AW, Cavanagh D (1990) Isolation of non-cytopathic viruses implicated in the aetiology of nephritis and baby chick nephropathy and serologically related to avian nephritis virus. Avian Pathol 19:139-160

7. Gibson CA, Chen J, Monroe SA, Denison MR (1998) Expression and processing of nonstructural proteins of the human astroviruses. Adv Exp Med Biol 440:387-391

8. Gough RE (1984) Laboratory confirmed outbreaks of duck virus enteritis (duck plague) in the United Kingdom from 1977 to 1982. Vet Rec 114:262-265

9. Harris C (2008) Turkey Coronavirus and Astrovirus in Britain. ThePoultrySite. http://www.thepoultrysite.com/

10. Imada T, Yamaguchi S, Mase M, Tsukamoto K, Kubo M, Morooka A (2000) Avian nephritis virus (ANV) as a new member of the family Astroviridae and construction of infectious ANV cDNA. J Virol 74:8487-8493

11. Jiang B, Monroe SS, Koonin EV, Stine SE, Glass RI (1993) RNA sequence of astrovirus: distinctive genomic organisation and a putative retrovirus-like ribosomal frameshifting signal that directs the viral replicase synthesis. Proc Natl Acad Sci USA 90:10539-10543

12. Jindal N, Patnayak DP, Chander Y, Zieglerr AF, Goyal SM (2010) Detection and molecular characterisation of enteric viruses in breeder turkeys. Avian Pathol 39:53-61

13. Koci MD, Schultz-Cherry S (2002) Avian astroviruses. Avian Pathol 31:213-227

14. Matsui SM, Greenberg HB (2001) Astroviruses. In: Knipe DM, Howley PM (eds) Fields virology, 4th edn. Lippincott Williams \& Wilkins, Philadelphia, pp 875-893

15. Maurel S, Toquin D, Men ML, Queguiner M, Eterradossi N (2009) Molecular epidemiology study of turkey astroviruses in France. In: Hafez HM (ed) Turkey production: toward better welfare and health. Berlin, pp 239-247

16. McNulty MS, Currran WL, McFerran JB (1980) Detection of astroviruses in turkey faeces by direct electron microscopy. Vet Rec 106:561

17. Nicholas RA, Goddard RD, Luff PR (1988) Prevalence of avain nephritis virus in England. Vet Rec 123:398

18. Pantin-Jackwood MJ, Spackman E, Woolcock PR (2006) Molecular characterization and typing of chicken and turkey astroviruses circulating in the United States: implications for diagnostics. Avian Dis 50:397-404

19. Pantin-Jackwood MJ, Spackman E, Woolcock PR (2006) Phylogenetic analysis of Turkey astroviruses reveals evidence of recombination. Virus Genes 32:187-192

20. Pantin-Jackwood MJ, Spackman E, Day JM, Rives D (2007) Periodic monitoring of commercial turkeys for enteric viruses indicates continuous presence of astrovirus and rotavirus on the farms. Avian Dis 51:674-680

21. Pantin-Jackwood MJ, Day JM, Jackwood MW, Spackman E (2008) Enteric viruses detected by molecular methods in commercial chicken and turkey flocks in the United States between 2005 and 2006. Avian Dis 52:235-244

22. Pantin-Jackwood MJ, Spackman E, Day JM (2008) Pathogenesis of type 2 turkey astroviruses with variant capsid genes in 2-day-old specific pathogen free poults. Avian Pathol 37:193-201 
23. Pantin-Jackwood MJ, Strother KO, Mundt E, Zsak L, Day JM, Spackman E (2010) Molecular characterization of avian astroviruses. Arch Virol 156:235-244

24. Reynolds DL, saif YM, Theil KW (1987) A survey of enteric viruses in turkey poults. Avian Dis 31:89-98

25. Saif LJ, Saif YM, Theil KW (1985) Enteric viruses in diarrheic turkey poults. Avian Dis 29:798-811

26. Smits SL, van Leeuwen M, Kuiken T, Hammer AS, Simon JH, Osterhaus AD (2010) Identification and characterization of deer astroviruses. J Gen Virol 91:2719-2722

27. Spackman E, Kapczynski D, Sellers H (2005) Multiplex real-time reverse transcription-polymerase chain reaction for the detection of three viruses associated with poult enteritis complex: turkey astrovirus, turkey coronavirus, and turkey reovirus. Avian Dis 49:86-91

28. Takase K, Murakawa Y, Ariyoshi R, Eriguchi S, Sugimura T, Fujikawa H (2000) Serological monitoring on layer farms with specific pathogen-free chickens. J Vet Med Sci 62:1327-1329
29. Tang Y, Ismail MM, Saif YM (2005) Development of antigencapture enzyme-linked immunosorbent assay and RT-PCR for detection of turkey astroviruses. Avian Dis 49:182-188

30. Tang Y, Murgia AM, Saif YM (2005) Molecular characterization of the capsid gene of two serotypes of turkey astroviruses. Avian Dis 49:514-519

31. Tang Y, Murgia MV, Ward L, Saif YM (2006) Pathogenicity of turkey astroviruses in turkey embryos and poults. Avian Dis 50:526-531

32. Villarreal LYB, Assayag MS, Brandao PE, Chacon JLV, Bumger AND, Astolfi-Ferreira CS, Jones RC AJF (2006) Identification of turkey astrovirus and turkey coronavirus in and outbreak of poult enteritis and mortality syndrome. Brasilian J Poult Sci 8:131-135 